\title{
Desigualdades Educativas Derivadas del Covid-19 desde una Perspectiva Feminista. Análisis de los Discursos de Profesionales de la Educación Madrileña
}

\author{
Educational Inequalities Derived from Covid-19 from a Feminist \\ Perspective. Analysis of the Discourse of Madrid Education \\ Professionals
}

\author{
Melani Penna Tosso * \\ Mercedes Sánchez Sáinz \\ Cristina Mateos Casado \\ Universidad Complutense de Madrid, España
}

\begin{abstract}
Se exponen y analizan los discursos de cinco profesionales de la educación, con formación en género y feminismo, en relación con las desigualdades educativas percibidas en el contexto urbano madrileño, ante la situación social y educativa generada por la Covid-19. Se realizó una investigación cualitativa a través de entrevistas semiestructuradas en base a bloques de contenidos desde un análisis naturalista de los discursos, empleando la triangulación. Las entrevistas fueron transcritas y analizadas buscando ideas compartidas desde una perspectiva feminista. Los resultados obtenidos permiten constatar la soledad de la labor docente durante estos meses de confinamiento, el abandono de la administración educativa, el aumento de un $30 \%$ en la dedicación horaria del trabajo, los problemas de salud física y salud mental entre las profesionales de la educación, la imposibilidad de continuar atendiendo las necesidades sociales y emocionales del alumnado ante la cantidad de contenidos curriculares a impartir y el olvido de la educación para la prevención de las violencias machistas. Las conclusiones principales vienen a constatar que el tiempo de trabajo ha tomado las casas, los espacios familiares y el tiempo libre y que se hace precisa una mayor coordinación y relaciones horizontales entre los equipos docentes aplicando políticas de cuidados. Será necesario poder atender emocionalmente al alumnado, así como realizar un cambio en el currículo para poder hacerlo compatible con los tiempos reales de la vida.
\end{abstract}

Descriptores: Covid-19; Educación; Desigualdades educativas; Género; Perspectiva feminista.

This paper exposes the educational inequalities perceived in the urban context of Madrid in the face of the social and educational situation resulting from Covid-19, by analyzing the discourses of five education professionals with training in gender studies and feminism. For the purpose of this qualitative study, we carried out interviews semi-structured around blocks of contents using a naturalistic discourse analysis approach and data triangulation. The interviews were later transcribed and analyzed from a feminist lens with the aim of identifying shared ideas between participants. The findings reveal the loneliness experienced by teachers during confinement, the impact of the abandonment of administrative work in educational institutions, a thirty percent hourly increase in the time spent working by education professionals, the physical and mental health issues faced by teachers due to the situation, the impossibility of continuing to meet the social and emotional needs of students in view of the amount of curricular content to be taught, and the lack of attention paid to gender-based violence prevention by educational institutions. The main conclusion drawn from this study is that working time has taken up homes, family spaces and free time and, as a result, there is an increasing need to implement improved coordination techniques, horizontal relationships between teaching teams, and care policies. It is necessary for teachers to be able to assist students emotionally, as well as to modify the curriculum in order to make it more compatible with people's life circumstances.

Keywords: Covid-19; Education; Educational inequalities; Gender; Feminist perspective.

*Contacto: melani.penna@edu.ucm.es

ISSN: 2254-3139

www.rinace.net/riejs/

revistas.uam.es/riejs
Recibido: 24 de mayo 2020

$1^{\text {a }}$ Evaluación: 17 de junio 2020

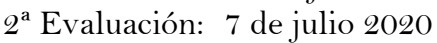

Aceptado: $\quad 20$ de julio 2020 


\section{Introducción}

La crisis social y sanitaria derivada de la Covid-19 ha generado en el ámbito educativo una situación sin precedentes. En las últimas semanas se han publicado diferentes entrevistas, investigaciones y artículos de divulgación general sobre esta temática. Sin embargo, en la gran mayoría de estas publicaciones relacionadas con lo educativo, no se ha reflejado de manera específica la labor del profesorado centrado en la atención a las diversidades, los principales problemas a los que tienen que hacer frente, las prácticas educativas que están implementando, ni las propuestas de cambio que considerarían importantes implementar en un futuro, aspectos todos ellos de especial relevancia para orientar al conjunto de agentes educativos ante esta situación, tanto a corto como a largo plazo (Gómez Gerdel, 2020).

Es igualmente relevante destacar, que en estas publicaciones han sido escasas las voces de las profesionales de la educación frente a las de los profesionales de la educación, invisibilizando la perspectiva de género que, tanto en este como en otros abordajes de las situaciones relacionadas con las desigualdades educativas, cobra especial relevancia (Simón, 2010).

Por todo ello, con la doble intención de situar las voces de las docentes y de la atención a las diversidades como un referente importante a la hora de describir la actual emergencia educativa, se diseñó esta investigación. Sus objetivos pueden concretarse en los siguientes:

- Especificar las principales dificultades percibidas por las profesoras y los departamentos y equipos de orientación, en relación con la atención a las diversidades en la actual situación de pandemia generada por la Covid-19.

- Exponer las prácticas educativas implementadas por dichas profesionales para disminuir las desigualdades.

- Visibilizar desigualdades de género que se dan en el ámbito educativo, relacionadas con la situación de pandemia entre el alumnado, el profesorado y las familias, desde una perspectiva feminista.

- Analizar las propuestas de cambio que proponen estas profesionales de la educación, ante posibles repeticiones de situaciones de emergencia similares.

\section{Revisión de la literatura}

En las bases de datos consultadas (ERIC, Compludoc, Dialnet, Teseo, ISOC, Google Schollar y Teacher Referente Center) apenas se encontraron publicaciones de investigaciones específicas que abordasen la repuesta educativa con una perspectiva feminista en la situación de pandemia. Por ello, la revisión de la literatura se centró en publicaciones relacionadas con la Covid-19 y la educación, ampliándose la búsqueda con artículos de opinión, noticias e informes.

Pasamos a resumir la revisión de la literatura dividida en los siguientes apartados: políticas educativas frente a la Covid-19, desigualdades educativas ante la Covid-19 y perspectivas de futuro desde la pandemia por Covid-19. 


\subsection{Covid-19 y políticas educativas}

Tal y como afirma Naomi Klein (Moreno, 2020) la pandemia de la Covid-19 parece haber llegado en un momento en el que la población desconfía de los dirigentes políticos y de sus capacidades para gestionar. El estado de crisis permanente en el que estamos desde hace años y que ha justificado durante este tiempo los recortes sociales, ahonda en el cansancio de la población y la desconfianza hacia la clase política (Santos, 2020).

Existen precedentes de políticas educativas desarrolladas en anteriores situaciones de pandemia como las derivadas del cierre de escuelas y centros educativos por la conocida como Gripe A (Cervantes y Gutiérrez, 2020) o las desarrolladas en relación con el Virus de Inmunodeficiencia Humana (Cejudo y Corchuelo, 2019).

Aunque las políticas estatales educativas ante la pandemia de la Covid-19 podrían parecer homogéneas, lo cierto es que en una comparativa de diferentes países, se aprecian importantes diferencias en cuanto al cierre de los centros educativos, el mantenimiento de la docencia online, la creación de comisiones de planificación educativa, el ajuste del calendario escolar y posposición de evaluaciones, los protocolos de seguridad, el tipo de reapertura y las actividades deportivas y los programas de apoyo individualizados (Zubillaga y Gortazar, 2020). Pese a estas diferencias, se puede afirmar que, a rasgos generales ante la pandemia actual, la mayoría de las políticas estatales han apostado, no sin polémica, por cerrar todos los centros educativos y continuar el curso mediante la docencia online (Almazán, 2020). Los sistemas de enseñanza parecen haberse adaptado rápidamente a estas circunstancias de cierre de centros (De Alonso, 2020; Rodríguez Morales, 2020) pese a no estar preparados para ello (Gárate, 2020) mediante una "enseñanza remota de emergencia" (Hodges et al., 2020).

Dentro de cada país, las políticas educativas que se están implementando frente a la pandemia son homogéneas, ignorando las desigualdades entre el estudiantado. Igualmente, las decisiones en materia educativa que se han ido adoptando desde las administraciones, se han hecho sin consultar al conjunto de agentes implicados, es decir, sin tener en cuenta los contextos educativos concretos en los que pretenden ser aplicadas con éxito (Rodriguez Morales, 2020; Gárate, 2020).

En un intento de sintetizar, Zubillaga y Gortazar (2020) destacan las siguientes medidas generales destinadas a atender la actual situación en el sistema educativo: ajuste curricular, reformulación curricular, evaluación global final, evaluación final diagnóstica, limitar la repetición de curso al máximo y planificar el seguimiento del alumnado con dificultades, desarrollo de programas específicos como las escuelas de verano, programas de refuerzo, planes específicos contra el abandono y el fracaso escolar, ajustes del calendario, provisión de recursos y establecimiento de medidas sanitarias preventivas y anticipatorias.

\subsection{Covid-19 y desigualdades educativas}

Tal y como afirma Boaventura De Sousa Santos (2020), las asimetrías se vuelven más visibles ante la pandemia. A nivel educativo se prevé, ante la actual situación, una disminución de la equidad (Peñate, 2020), un aumento de las desigualdades (Gómez Gerdel, 2020), un aumento del abandono escolar -que en algunos países como España ya era elevado (De Alonso, 2020)- y problemas económicos en las familias, especialmente en las familias pertenecientes a colectivos en riesgo de exclusión social (Juárez, De Oña y Molina, 2020). 
Se denuncia la falta de apoyo al profesorado por parte de las administraciones, la falta de coordinación y la tendencia a culpabilizar al profesorado (Gárate, 2020). Igualmente se señala el riesgo de la burocratización de la labor docente (Almazán, 2020), frente al papel esencial que deberían tener en estas circunstancias el trabajo en equipo y la coordinación (Muñoz y Lluch, 2020), así como el trabajo de los departamentos y equipos de orientación (Gómez Gerdel, 2020).

La comunidad educativa no estaba preparada para asumir las labores de docencia en la distancia, tal y como han sido planteadas a partir de la situación de confinamiento (Peñate, 2020). Además, lejos de facilitar la labor educativa, la gran cantidad y variedad de recursos disponibles la ha dificultado, tanto al profesorado, como al alumnado y las familias, que no han sabido seleccionar ni organizar estos recursos (Zubillaga y Gortazar, 2020).

La continuidad de las clases mediante la docencia online está generando desigualdades. En este sentido se calcula que más de ochocientos millones de menores no tienen acceso a un ordenador desde sus casas. Esta situación afecta de manera desigual a los diferentes países (Gascón, 2020), y dentro de los diferentes países hay diferencias en función del entorno (Zhong, 2020). En el estado español, UNICEF calcula que más de cien mil hogares no tienen acceso a internet (Gascón, 2020).

Igualmente se han constatado diferencias entre los centros educativos en su capacidad para adaptarse a la docencia a distancia. Estas diferencias están relacionadas con múltiples factores, como el hecho de contar con una plataforma online eficaz de apoyo a la enseñanza. En este sentido, en investigaciones anteriores (INE, 2019) se constata que menos de la mitad de los equipos directivos de los centros públicos del territorio español considera que su centro contaba con este recurso.

Las desigualdades entre el alumnado también han aumentado. El estudiantado con escasos recursos de zonas urbanas presenta graves dificultades para mantener las clases a distancia, sin las herramientas necesarias y teniendo un mayor riesgo de contagio (Rodríguez Morales, 2020). La inseguridad laboral de las familias se ha agudizado durante esta pandemia y se prevé que aumentará, afectando al alumnado en su rendimiento (RuizValenzuela, 2020), aunque en este punto se requiere un análisis interseccional y pormenorizado de las diferentes situaciones (De Sousa Santos, 2020).

Junto a estas dificultades ha aumentado la carga de tareas que tiene que realizar el estudiantado en casa (Corres-Medrano y Santamaria-Goicuria, 2020) sin modificar los contenidos a enseñar. Este aumento en la carga de tareas puede estar generando, especialmente en el alumnado con dificultades de aprendizaje, un estado emocional negativo (Ceballos y Sevilla, 2020).

Aunque es poco lo que se sabe del alumnado que no se está conectando a la docencia online (Rodríguez Morales, 2020), sí se puede afirmar que la dificultad para continuar este modelo educativo a distancia es mayor en el alumnado de las primeras etapas educativas, por ejemplo, en la Comunidad de Madrid, la dificultad para seguir la enseñanza online afecta al 30\% del alumnado de Educación Primaria, frente al 22\% del alumnado de Educación Secundaria y el 10\% del alumnado de Bachillerato (Comunidad de Madrid, 2020).

Zubillaga y Gortazar (2020) tratan de predecir la pérdida en el aprendizaje que supondrá este cierre de las escuelas, adaptando los resultados de la investigación de Copper, Borman y Fairchild (2010) sobre el impacto del verano en el aprendizaje. Si esta comparación fuera 
correcta, se calcula que el alumnado con nivel adquisitivo medio y alto perderá un $20 \%$ del aprendizaje, mientras que el alumnado de nivel adquisitivo bajo, desaprenderá un $50 \%$ de los contenidos abordados durante la parte del curso presencial.

En relación con las desigualdades educativas, igualmente se debe tener en cuenta que la violencia machista se ha intensificado con el confinamiento (Gil, 2020), afectando gravemente a las dinámicas familiares y educativas. Según el Informe La perspectiva de género, esencial en la respuesta a la Covid-19 (Ministerio de Igualdad, 2020), el confinamiento ha supuesto un aumento del riesgo de violencia de género, registrándose un incremento del $60 \%$ en las peticiones de asistencia a víctimas de violencia de género, en relación con el mismo periodo del año anterior. Insiste el Informe en que ignorar el impacto de género en la situación causada por el coronavirus, tendría consecuencias económicas y sociales que agravarían la desigualdad.

\subsection{Covid-19 y perspectivas educativas de futuro}

La actual situación desencadenada por la Covid-19 puede transformarse en el ámbito de la enseñanza en una oportunidad educativa (Cervantes y Gutiérrez, 2020; De Alonso, 2020; Gómez Gerdel, 2020) para estudiantes, docentes y familias, si se sabe aprovechar el momento para recuperar tiempo propio y realizar un aprendizaje más centrado en el entorno cercano que posibilite frenar las desigualdades de género (Díez-Gutiérrez, 2020), aumentando el tiempo para reflexionar sobre el impacto que la digitalización va a tener sobre la labor docente (Almazán, 2020).

Los centros educativos van a necesitar recursos para compensar las pérdidas que la situación actual ha generado en la enseñanza (Burgess y Sievertsen, 2020). Sobre el tipo de recursos y apoyos concretos que se van a requerir, existen discrepancias relacionadas con divergencias profundas respecto a diferentes propuestas políticas (Fernández Amador, 2020) y educativas que subyacen a estas iniciativas. Algunas investigaciones plantean que la desigualdad educativa generada por esta crisis puede ser compensada si se implementan las medidas apropiadas, tales como apostar por la educación pública, la inversión educativa y la reducción de las ratios (Díez-Gutiérrez, 2020).

Ante esta situación es importante ser capaces de imaginar otras realidades a las que queramos regresar (Berardi, 2020; Gil, 2020; Preciado, 2020), desarrollando una "imaginación preventiva" (Santos, 2020) y cuestionando la intensificación actual del trabajo escolar que puede aumentar las presiones y el estrés, aspectos contraproducentes para cualquier proceso de aprendizaje en profundidad, tanto para el profesorado, como para el alumnado y las familias (Díez-Gutiérrez, 2020).

Cuando las crisis se vuelven algo permanente. pueden utilizarse, como ha ocurrido con las crisis económicas desde el año 2008, para justificar cualquier tipo de medida que se adopte (De Sousa Santos, 2020). Para que esto no ocurra, desde postulados feministas, se plantea la necesidad de que esta crisis social, sanitaria y educativa coloque en el centro del debate las ideas feministas (Díez-Gutiérrez, 2020; Gil, 2020), los cuidados y el papel de las mujeres (Bassets, 2020), el eco-feminismo y un papel activo de la ciudadanía, planteando preguntas y opiniones, compartiendo y debatiendo ideas (Fernández Savater, 2020). Si como modelo social y educativo, el capitalismo no tiene futuro, resulta pertinente preguntarse qué nuevo modelo social y educativo pretendemos (Santos, 2020). 


\section{Método}

La situación de estado de alarma en la que se desarrolló la investigación restringió sus posibilidades metodológicas. La metodología empleada fue cualitativa y explicativa. Se centró en analizar en profundidad las ideas y opiniones de una muestra reducida de profesionales de la educación mediante el análisis crítico de sus discursos (García Medina et al., 2020).

Tras una primera categorización, desde una perspectiva de investigación feminista (Anzaldua, 2004), se pusieron en diálogo, y no en confrontación, las ideas planteadas por las diferentes profesionales, tratando de tejer un discurso común (Sandoval, 2004), que se expone en el siguiente apartado, como resultado de la investigación.

Frente al sonambulismo tecnológico (Almazán, 2020) y la proliferación de estudios cuantitativos que buscan recoger la opinión general de una muestra amplia de la población, esta investigación buscó visibilizar las voces críticas de las docentes con perspectiva feminista, analizando sus discursos sobre la atención a las desigualdades, buscando ampliar un debate, el del futuro de la educación en el siglo XXI ente la pandemia de la Covid-19, que parece estar anclado en posiciones dicotómicas (Gárate, 2020).

Plan de muestreo

Se optó por un muestreo no probabilístico intencional mediante estudios de caso representativos. La población objeto de estudio fueron mujeres: docentes, orientadoras, educadoras sociales y personal técnico de servicios a la comunidad, que estaban trabajando en las etapas de educación obligatoria en centros públicos de la Comunidad de Madrid y que tenían formación en género y feminismo.

La muestra final estuvo constituida por un caso representativo de cada uno de los sectores vinculados de manera relevante con la atención a las diversidades. En total fueron cinco profesionales de la educación $(\mathrm{N}=5)$ con los siguientes perfiles:

- Una orientadora escolar perteneciente a un Equipo de Orientación Educativa y Psicopedagógica.

- Una personal técnica de Servicios a la Comunidad.

- Una educadora social.

- Una tutora del Programa de Mejora del Aprendizaje y el Rendimiento.

- Una profesora de Educación en Valores.

La muestra global estuvo constituida por mujeres con edades comprendidas entre los 35 y los 50 años y una experiencia laboral que oscilaba entre los 5 y los 20 años de trabajo en el ámbito educativo en centros educativos públicos y/o asociaciones. Todas ellas tenían formación de posgrado en género. Cuatro de las cinco personas entrevistadas eran activistas y formaban parte de grupos feministas de trabajo en torno a lo educativo.

Instrumento utilizado

Se pretendía recoger y analizar el pensamiento crítico de estas profesionales, por lo que se consideró pertinente el uso de una entrevista semiestructurada, elaborada ad-hoc. 
Se incluyeron preguntas en relación a los datos sociodemográficos, así como cuestiones relativas a los siguientes bloques temáticos, considerados de interés en función de los objetivos de la investigación y la revisión de la literatura.

- Situación personal, familiar y de salud de la profesional: salud mental y física de la docente, situación de salud de familiares, responsabilidades y cuidados.

- Situación laboral: espacio de trabajo, horas de dedicación, funciones que está desempeñando, perfil del estudiantado a su cargo.

- Labor docente: objetivos, apoyos, recursos, buenas prácticas

- Género y confinamiento: género y desempeño del alumnado y del profesorado, brecha de género y pandemia.

- Aprendizajes para futuras situaciones similares: aprendizajes y cambios.

\section{Procedimiento}

Las entrevistas fueron realizadas por video-llamada en un horario convenido con la docente. Fueron grabadas y posteriormente transcritas. Tuvieron una duración media de 53 minutos. Al inicio de cada entrevista se grabó el acuerdo de consentimiento informado.

\section{Análisis de datos}

Los datos fueron analizados a partir de los bloques de contenidos sobre los que se recogió información. En los siguientes apartados donde se redactan los resultados, se han incluido algunos de los fragmentos más representativos de las entrevistas, en los que hubo más consenso. Cada uno de los fragmentos, se encuentran identificados con las iniciales mayúsculas del perfil profesional de la docente.

\section{Resultados}

Los resultados se exponen siguiendo los bloques de contenido de las entrevistas.

\subsection{Situación personal, familiar y de salud}

Todas las docentes entrevistadas, la primera pregunta que hacen cuando escuchan si se encuentran bien de salud es “ffísica o mental?", haciendo distinción entre ambas y concluyendo que físicamente bien pero no mentalmente. Coincidiendo en general con que se encuentran bien físicamente, el malestar psicológico y emocional se hace evidente y se instala inmediatamente en el discurso con expresiones como: "me cuesta estar bien", "dispersa", "con poca capacidad de concentración", con "problemas para dormir", "revuelta", "agotada".

La mayoría de las profesionales entrevistadas no tienen familiares a su cargo con necesidades especiales de atención o cuidado. De las profesionales entrevistadas, una de ellas tiene hijos menores y madre y padre en situación de vulnerabilidad, lo que ha supuesto una doble responsabilidad, y otra, manifiesta haber tenido que desplazarse a la vivienda familiar a cuidar de sus familiares mayores.

\subsection{Situación laboral}

Las profesionales entrevistadas ocupan los puestos especificados anteriormente. Todas ellas, excepto una, tienen contratos como funcionarias, en plazas con destinos temporales o definitivos. Las peores condiciones y el mayor grado de inestabilidad laboral lo registra 
la educadora social, vinculada a un programa de apoyo psicosocial y educativo en dos IES del centro de Madrid.

Todas las profesionales entrevistadas se encuentran trabajando de manera activa y la situación de confinamiento ha supuesto un aumento de sus jornadas laborales, con un incremento semanal de la dedicación que oscila entre las 9 y las 15 horas.

La modalidad de teletrabajo por la situación de confinamiento les ha impuesto la obligación de estar híper conectadas, debido a la integración y adaptación al sistema de las primeras semanas, a la falta de coordinación en los centros educativos y a la presión de estar siempre dispuestas a resolver dudas del alumnado con necesidades especiales y de familias en situación de vulnerabilidad (que han empeorado su situación con el estado de alarma). Un claro ejemplo de esto es como la utilización del teléfono personal para las llamadas al alumnado permite tener acceso a entrar en contacto con el profesorado a cualquier hora:

Si recibo algún mensaje de algún chaval o alguna chavala fuera del horario de trabajo
pues lo voy a contestar (...) el viernes por la noche tuve que hablar con un chaval
porque había tenido en casa una historia tremenda entonces estuve todo el fin de
semana hablando con él (...). Te escriben a las siete y media de la tarde preguntándote
si les echas una mano con las tareas y lo haces. (ES)

Los lugares de trabajo son valorados por las entrevistadas como poco cómodos, inapropiados, insanos, ya que consideran que el espacio de trabajo se confunde con el espacio de la vivienda y de la habitación en casas pequeñas, con la obligación de conciliar y compartir el mismo espacio con otras personas. El mobiliario resulta inadecuado para jornadas de trabajo muy largas, lo que está provocando problemas físicos, principalmente en piernas y espalda, que no son nombrados como tales en respuesta a la primera pregunta.

Todas las profesionales entrevistadas trabajan con sus propios recursos tecnológicos. La institución para la que trabajan en ningún momento se ha puesto en contacto con ellas para valorar las dificultades del teletrabajo desde sus hogares: "el otro día la directora me preguntaba, después de casi dos meses, ¿̇oye, tú tienes tarifa ilimitada de minutos?" (PTSC)

Trabajan con un volumen de alumnado que oscila entre los 21 y 207 estudiantes, algunos de ellos con necesidades educativas especiales (NEE). Un gran número tiene problemas sociales y económicos, nivel cultural y rentas bajas, no tienen recursos tecnológicos para poder conectarse, ni tampoco las habilidades necesarias o el interés y la importancia al estudio que requiere. Por otro lado, opinan que el alumnado de clase media, por lo general, no tiene dificultades de conectividad y tienen tecnología a su disposición para poder acceder al estudio online. Existe una importante brecha digital, con familias que no tienen acceso a dispositivos tecnológicos o de conexión, para los que se han adherido a proyectos propios de cada IES, de la Comunidad de Madrid o incluso de Cruz Roja u otras instituciones, para que accedieran a una Tablet o a conexión a Internet, ayudas que han sido escasas y han llegado tardíamente.

El confinamiento educativo ha venido a complicar la situación de una parte del alumnado que, ya en la educación presencial, era potencialmente absentista o resultaba difícil de motivar:

Hay otra parte del alumnado que ya estaba desconectado en las clases presenciales (...) estaba perdido académicamente hablando y que el contacto presencial permitía poderlos enganchar y poderlos animar (...). Más o menos el 15\% de mis estudiantes está en esta situación. Este desinterés no viene de la nada, viene muchas veces de un 
contexto familiar que no les motiva para nada a estudiar y si ahora están todo el día en casa... (VAL)

Los alumnos que ya eran absentistas desaparecen (...). Están totalmente desvinculados de lo académico. Yo solo llamo para saber cómo están, pero tareas no hacen. Los profes dicen que no ha hecho nada en todo el año y no quieren gastar la energía con ellos. Entonces solo quedo yo llamando. (PTSC)

La distancia y la dificultad de conexión de parte del alumnado han dificultado mucho, sobre todo las primeras semanas, el proceso de enseñanza-aprendizaje. La mayor parte del alumnado que ha tenido dificultades con la conexión y con seguir el ritmo del curso en situación de confinamiento, es alumnado con NEE:

\begin{abstract}
Pues no he podido contactar, en porcentaje, fácil con un 10\% de la clase. Les he escrito por correo electrónico, he llamado a los teléfonos de contacto que tenemos y he intentado hablar con las tutoras y ha habido varios casos en los que no ha habido forma de contactar de ninguna manera y ha habido otros en los que, cuando hemos contactado, se nos ha dicho que la situación era muy complicada por un motivo u otro y que la conexión iba a ser claramente intermitente o ausente. (PMAR)
\end{abstract}

Con las familias también se han presentado serios problemas de comunicación. Tras mucho insistir, se ha logrado establecer contacto con la gran mayoría. En algunos casos se ha planteado por el nivel de desconexión de las familias "que vaya el agente tutor o la agente tutora a casa porque hay familias que no se sabe nada de ellas" (OE).

\title{
4.3. Labor docente
}

La labor docente ha tenido dos periodos diferenciados, uno que va desde el decreto de estado de alarma hasta las vacaciones de semana santa y otro posterior con la estabilización del estado de alarma y la desescalada.

El primer periodo, de manera general, ha estado marcado por problemas relacionados con la falta de coordinación institucional, la soledad de las profesionales, las dificultades de organización laboral, unidas a las necesidades de organización doméstica y familiar, y los problemas tecnológicos:

Me he visto teniendo que reorganizar la máquina estando sola, porque para mí uno
de los problemas fundamentales de esta situación es la soledad. Era una especie de
tsunami. Era cambiar todo sabiendo que había mucha gente que te está esperando. En
mi centro no ha habido ninguna coordinación era "vete a tu casa, no sabemos lo que
va a pasar y apánatelas como puedas". Después de tantos años en esto me he visto muy
poco provista de herramientas, desprotegida y sola. (VAL)

Los objetivos que se planteaban, más que educativos, fueron emocionales. Entraron en contacto directo con el alumnado vía telefónica, especialmente con aquel con NEE, para saber si se encontraban bien, cuál era su estado de salud y el de su entorno, cómo estaba la relación con sus familias, y si disponían de la conexión y la tecnología precisas. Las primeras semanas, se podrían resumir con un "hola, estoy aquí, no me he ido" (ES), concentrando al máximo el propósito educativo en la presencia cotidiana en modo virtual:

El principal objetivo que me he marcado desde el principio como tutora era tener, al menos semanalmente, el contacto con mis alumnos vía telefónica para saber si se encontraban bien, cuál era su estado de salud, si tenían alguna necesidad... más allá de lo académico, para poder tratar de ayudar. (PMAR)

Quería que mi alumnado se sintiera cerca, supiera que podía confiar en mí y todo esto como una responsabilidad y un servicio público (VAL). 
Se ha prestado especial atención en esta fase al acompañamiento al alumnado y a sus familias, generando material divulgativo para las familias:

Hemos ido facilitando material que nos solicitaba los centros, como material de refuerzo para los niños y las niñas, adaptado a las características de nuestras familias, que no les podemos mandar un material cualquiera, muy técnico o muy poco accesible. (OE)

Otro de los objetivos iniciales fue capacitar al alumnado para poder enfrentarse a la enseñanza online:

\begin{abstract}
Al principio, los problemas fundamentales a los que te enfrentas es cómo hacerle llegar a todo un alumnado (...) que tenían un usuario y una contraseña para entrar en el aula virtual y cómo explicarles, desde la distancia, y teniendo en cuenta muchas veces las situaciones de dificultad de conexión desde sus hogares y de falta de dispositivos. Esto para quienes contestaban al teléfono y a los que les podías hacer llegar esta información de la plataforma que ibas a utilizar, pero había muchos que no teníamos sus direcciones de correo electrónico, y si las teníamos no contestaban a ellos o no sabían utilizar la información que les mandábamos, entonces esto supuso en las primeras semanas muchas horas de llamadas de teléfono. (PMAR)
\end{abstract}

Los objetivos de la segunda fase se han centrado más en lo académico. Se comenzaron a recibir instrucciones más precisas y las entrevistadas reconocen una readaptación de objetivos. En esta fase se detectó que el alumnado tenía muchas dificultades "no solo para entender la tarea que se les asignaba sino para organizar todas las tareas que se les mandaban desde diferentes asignaturas" (PMAR). La sobrecarga en las tareas ha sido una percepción generalizada:

Ahora estamos en cómo afrontamos los deberes, los talleres de técnicas de estudio que ahora han cambiado por la tecnología y por no tener a la profesora al lado (...). Otra dificultad que he encontrado es que muchos de ellos no tenían los libros (...). Ahora la demanda ha crecido mucho en torno a los deberes. Ha habido mucha historia con lo de la evaluación, esto les ha generado mucha ansiedad. (ES)

El espacio educativo formal ha entrado en relación directa con el espacio familiar y esto ha permitido analizar más de cerca las demandas familiares y reorganizar los objetivos planteados inicialmente. Se evidencia, de manera mucho más clara, falta de apoyos familiares, debilidades y brechas compartidas entre el sistema educativo y el familiar, pero, por otro lado, se identifican beneficios relacionados con el aumento del tiempo familiar:

Muy poco apoyo en casa, entonces esta labor de organizar las tareas de nueve asignaturas diferentes y poder llevarlas al día, muchos de ellos tenían muchas tareas retrasadas, entonces ahi cambia el objetivo. No cambia tanto el que el objetivo sea tratar de acompañarles para que puedan sacar adelante las máximas asignaturas, sino que cambia la forma. (PMAR)

No tienen espacio, no tienen intimidad, entonces a la hora de hablar es difícil. Hay menores que la comunicación solo va por el WhatApp. Estoy haciendo video llamadas con cuatro y tengo 21, muchas de ellas no quieren porque no tienen intimidad, no tienen espacio, no tienen ordenador. (ES)

Algunas alumnas y alumnos que tenían una situación muy difícil y he visto que, el hecho de estar confinados y estar con sus familias, les ha permitido despuntar, que han encontrado un apoyo y un afecto que les han podido proporcionar porque nadie podía salir de casa y tenían disponible ese tiempo que antes laboralmente no tenían, esto creo es una cosa que se ha ganado en algunos casos, en otros casos ha sido, al contrario. (PMAR)

Reconocen que los objetivos señalados en un principio se fueron modificando con el paso de los días y la realidad, dejado a un lado proyectos y priorizado las necesidades del día a 
día y los seguimientos educativos y afectivos que permitían reforzar el vínculo y compromiso educativo:

Al principio hice un curso de cómo crear un blog, pero no está sirviendo de mucho. Hice un curso, lo cree, pero ahora está apartado porque vas dando respuestas más concretas y específicas para cada familia. (ES)

Al principio me organicé las tareas que iba a hacer en las dos primeras semanas, organicé un proyecto de habilidades sociales, también pensé un programa con los alumnos mediadores. Hice seguimiento y pensé actividades para los alumnos absentistas. Para ver cómo se podían reincorporar a la vuelta. También el seguimiento de alumnos que yo sé que tienen problemas en casa. Ahora me paso las semanas llamando para hacer seguimiento. El proyecto de habilidades sociales y lo de los delegados de convivencia lo he dejado a un lado y priorizo las necesidades del día a día, y para que no se desvinculen. (PTSC)

Entre los problemas generales señalados, se encuentran la descoordinación por parte de las autoridades de la Consejería de Educación de Madrid, unido a una filosofía institucional de seguir funcionando en una normalidad aparente e impuesta. Se mantuvieron los objetivos marcados, a pesar de que el nuevo contexto no estaba dando respuesta a una educación en situación de emergencia, pero sí a la justificación del trabajo docente remunerado y a la burocracia administrativa.

Parecía que todo el mundo teníamos que estar como trabajando "normal" cuando no era una situación normal. (OE)

Hay muy poca coordinación como equipos docentes y las labores están atomizadas, cada profesor utiliza una plataforma diferente. (PMAR)

De igual manera la coordinación departamental ha sido compleja "no siempre la gente está dispuesta o no entienden el trabajo coordinado de la misma manera" (VAL). Manifiestas que buena parte del tiempo, ha tenido que ser dedicada a generar una cultura de equipo que no existía.

Tanto jefatura como dirección, cuando he tenido alguna consulta directa y clara que hacer y plantear me han respondido y me consta que están trabajando muchísimo (...) y lo mismo con mi jefatura de departamento. Pero creo que las labores de coordinación de estos órganos han brillado por su ausencia, yo las he echado mucho de menos, he echado de menos una actitud más proactiva, que fueran estos órganos de coordinación quienes coordinaran, propusieran actividades de coordinación, no solo respondieran a dudas, sino que hubieran planteado determinados espacios para intercambiar y salir de esta lógica atomizada. (PMAR)

En cuanto a los apoyos, de manera general, se han sentido bastantes solas, con poco apoyo por parte del equipo directivo o de la Administración para afrontar la emergencia educativa.

De la administración no ha habido ningún tipo de apoyo y sigue sin haberlo, nos dan instrucciones contradictorias (...) nada de apoyo. (OE)

Simplemente nos mandan órdenes, nos mandan instrucciones que debemos de responder, la mayoría de ellas son burocráticas y, en este sentido, me he sentido maltratada por la propia administración y por el equipo directivo que responde a las órdenes de la administración. (...) Entonces me he sentido como una especie de monigote a disposición de una administración que no se preocupa por lo humano y, por otro lado, teniendo claro que, independientemente de esto, por encima de todo está mi alumnado, por lo que estoy intentando que puedan acabar el curso, seguir educando, aunque sea online. Entonces en lo personal con el alumnado estoy ahí, pero con la administración estoy muy desconcertada y frustrada. (VAL) 
Todas las profesionales coinciden en que han tenido escasos apoyos institucionales y que el único apoyo reconocido y continuado ha sido el de sus redes personales y el trabajo en equipo. Las redes profesionales también han sido un gran sostén. Hay unanimidad en considerar que lo que ha funcionado son las relaciones humanas y de equipo por encima de todo. El apoyo mutuo, la flexibilidad, la adaptación:

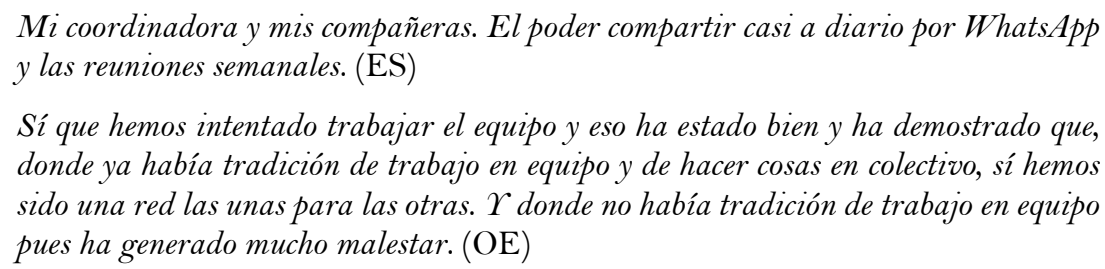

En cuanto a los recursos, una dificultad ha sido manejar los problemas técnicos, sociales y económicos, al mismo tiempo que la motivación. Algunos de los estudiantes ya se encontraban desmotivados en condiciones habituales y el contacto físico era fundamental para mantener el ánimo. En el caso de este alumnado, señalan que ha sido fundamental las llamadas uno a uno para conseguir una respuesta activa.

En cuanto a los medios de comunicación, ha funcionado, más que el uso del correo electrónico, llamar a través del móvil o del WhatsApp. Las vídeo llamadas individuales y grupales se han revelado efectivas. Las herramientas facilitadas por la Comunidad de Madrid como el aula virtual también han funcionado.

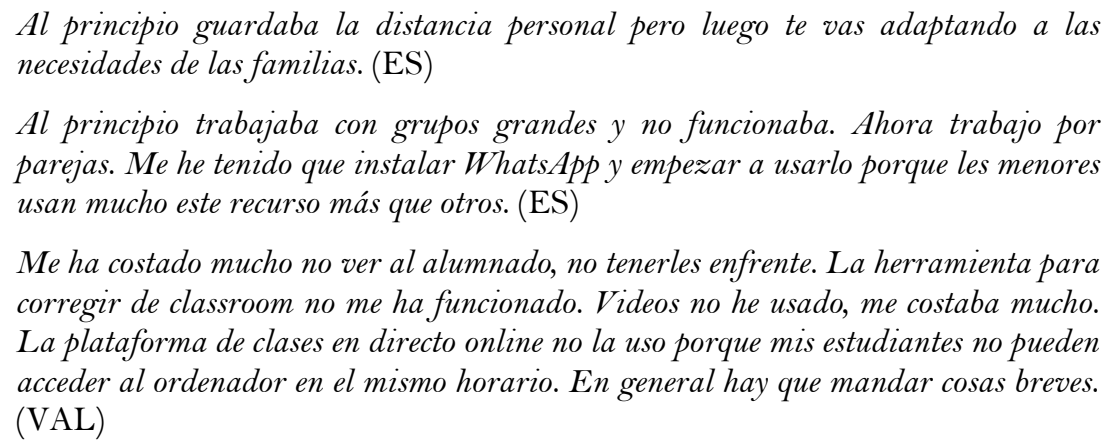

Me ha costado mucho no ver al alumnado, no tenerles enfrente. La herramienta para corregir de classroom no me ha funcionado. Videos no he usado, me costaba mucho. La plataforma de clases en directo online no la uso porque mis estudiantes no pueden acceder al ordenador en el mismo horario. En general hay que mandar cosas breves. (VAL)

Ha sido necesario usar diferentes formatos para no aburrir al alumnado (lecturas, imágenes, audios, noticias actuales...). Ponerse en contacto de manera personal ha sido fundamental ya que las redes de grupo no han dado respuesta a la situación y en ocasiones han generado conflictos y discusiones internas difíciles de manejar por el profesorado.

Han funcionada las tutorías, las video conferencias y las llamadas personales, el estar ahí, y esto es lo más parecido a la docencia presencial, porque les estás viendo y te están viendo y estás charlando de tú a tú. Este es el recurso con el que me siento que realmente sé cómo están, saben cómo estoy y sé lo que tienen que hacer, si tienen dificultades pues solucionas en el momento. (PMAR)

También enfatizan la importancia de la coordinación permanente y señalan como fundamental permitir que el alumnado exprese lo que siente y lo que está viviendo.

No destaca de manera general la identificación de buenas prácticas. Sí, algunas cuestiones de la organización como "el trabajo horizontal con los equipos de apoyo de los coles, sin protagonistas en los procesos y todo basado en la colaboración y el apoyo mutuo" (OE). Y de manera concreta algunas herramientas como "las guías de recursos accesibles a través de la web de los IES" (PTSC), o realizar actividades para "poder hablar cómo se sienten con lo que está pasando" (VAL). 
En un futuro prevén problemas relacionados con la accesibilidad a las familias, realizar los trámites burocráticos y con "un aluvión de reclamaciones de estudiantes que ya iban mal en el curso en los dos primeros trimestres" (VAL). Proyectan que va a ser un inicio de curso complicado y consideran que habrá estudiantes afectados profundamente por las consecuencias sociales de la Covid-19 y que no van a tener atención específica. Apuntan con preocupación a que se debería estar reorganizando el trabajo y tomando decisiones "y eso va a condicionar la atención a la diversidad el año que viene” (OE).

\subsection{Educación, género y confinamiento}

De manera generalizada, consideran que las cuestiones de género en el ámbito educativo para la administración, el equipo directivo o los departamentos ya eran bastante poco tenidas en cuenta e incluso, irrelevantes, cuestión que ha empeorado:

El análisis desde una perspectiva de género ha sido borrado. (OE)

No ha habido ningún tipo de atención ni hay nada. (...). Desatención a las cuestiones de género en general (...). No son una prioridad ni algo que se plantee (...) las cuestiones de género son irrelevantes. (VAL)

Alguna de las profesionales manifiesta que, en lo primero que pensó, fue en las alumnas que tienen situaciones de violencia intrafamiliar y en el posible agravamiento de su situación. En sus análisis de género como profesionales, sobre el impacto de la crisis sanitaria y social en el alumnado, destacan que las alumnas, de manera general, han seguido manteniendo un buen rendimiento a pesar de estar expuestas y presentar mayores problemáticas socio familiares, una mayor presión y responsabilidad en los cuidados y menor acceso a la tecnología:

To he notado que chicas que entregan tareas con retraso porque tienen que hacer más tareas en casa además de sus tareas del instituto. (VAL)

Tengo cuatro chavalas que cada vez que hablo con ellas están con los hermanos pequeños, son ellas las que cuidan. $Y$ luego tengo bastantes familias monoparentales o separadas y ahi sí que el peso lo tienen las mamas. (ES)

Tengo una alumna que solo entrega las tareas cuando está con la madre, lo que hace pensar que cuando vive con el padre se encarga ella de las labores de cuidado. (PMAR)

En el tema de cuidados, del acceso a los dispositivos móviles, de ejercicio de poder para usar el ordenador "quien usa el ordenador", eso se está reproduciendo en las casas sin duda. $(\mathrm{OE})$

Por otro lado, se ha manifestado una alarmante desconexión académica de algunos chicos por ocupar buena parte del tiempo en los juegos online.

También hay problemas de adicciones a los juegos. $Y$ esto genera una desorganización de horarios, que se levantan a las tres de la tarde y se pasan toda la noche jugando a la Play o a juegos online. Lo de la Play es clave, los que juegan a la Play son los chicos, se tiran horas. $Y$ he visto en algunos chicos, hablar con ellos y solamente remitirse al juego que están jugando de la Play. (ES)

En cuanto a la diversidad y las relaciones de género en la comunicación y gestión de los grupos, se pueden analizar amplias diferencias basadas en la discriminación de género, los diferentes roles asignados, el machismo y la violencia internalizada.

El chico que ha fallecido su abuela no ha querido compartir nada, no ha querido hablar de nada y ellas han tenido una demanda mucho más profunda y de unión, entre ellas si han querido hacer video-llamadas, vernos. Y eso lo veo como una fortaleza de las chicas. (ES) 
Como usan el chat ellos y ellas no tiene nada que ver. Ellos mandan videos violentos y ellas son las que tienen que poner el freno, de "oye, este grupo no está para esto, este es un grupo para cuidarnos". Una chica de uno de los grupos se ha salido porque compartían todo el tiempo videos de play. (ES)

Se muestra especial preocupación y se señala como un problema grave el alumnado con situaciones de violencia intrafamiliar y de situaciones de acoso por el uso masivo de las redes. La situación de confinamiento complica en gran medida, según las profesionales, la detección y la intervención en estos casos.

Estas situaciones muchas veces cuando las detectas es por el contacto personal, porque hay un momento de intimidad con el alumnado, con una determinada chavala, en un pasillo, al terminar la clase... le ves una cara y entonces le pides que se quede, le preguntas... todo esto desde una pantalla, no lo haces, no lo identificas, no sabes si está pasando. Hay muchas veces que esta detección tiene que ver con rasgos súper sutiles (o no tanto, a veces son físicos) que identificas en la presencia y los estás viendo, pero no los ves a través de estos medios digitales. Esto es fundamental. (PMAR)

Consideran que, frente a algunas familias y hogares, el espacio educativo se presenta para parte del alumnado como el único espacio de equidad.

Hay para niñas que el cole es un contexto de equidad y la casa no. (ES)

El cole es un contexto de equidad para muchas niñas y la casa no, independientemente de la estratificación social o los ingresos o la raza o la etnia o su familia, lo que en el cole detectamos que en las casas no hay tareas equitativas pues ahora está pasando.

$(\mathrm{OE})$

En al análisis de género desde los equipos profesionales, se ha notado la brecha de en el reparto y la asunción de las tareas. Las profesoras "llaman más y están más preocupadas por la situación que suele tener el alumno (...) están mucho más en tareas académicas a la par que en tareas emocionales" (ES). En general opinan que los profesores han asumido en exclusividad las tareas de competencia digital.

Los tíos se están encargando mucho de la parte digital, de competencia digital, de organizar los meets y las tías estamos como más a otras tareas de lo emocional. (OE)

Aseguran que estas y otras violencias, basadas en estereotipos, prejuicios y roles sexistas ya estaban en los equipos, pero el confinamiento ha revelado un ambiente "pasivoagresivo en las reuniones, y la gente más irascible”. (OE)

En cuanto a la corresponsabilidad, si existía de manera nítida, ahora aseguran que ha desaparecido.

Las compañeras tienen el doble de cuidados que hacer en casa y la administración le exige como sifueran máquinas de trabajar, solo ven que son profesionales y no madres y cuidadoras. (VAL)

Las profesoras también están con la conciliación y suelen llegarme llamadas o WhatsApp por las tardes, no por las mañanas, me imagino que por la conciliación.

(ES)

\subsection{Aprendizajes para futuras situaciones similares}

Manifiestan de manera unánime que han sentido el juicio negativo y la presión social por justificar su trabajo y su salario, lo que ha llevado a asumir de manera general un sistema de burocracias y sistematizaciones que contempla al alumnado de manera homogénea, sin tener en cuenta sus especificidades. Se presenta también una crítica docente generalizada frente al envío masivo de tareas, considerando que también ha sido provocado, más que por una falta de coordinación, por la presión del trabajo docente frente a la administración: 
Mi hija está en infantil y el primer WhatsApp de un papa cuando empezó lo del confinamiento fue "los profes de vacaciones y yo qué hago con mi hija", ese comentario refleja cómo nos ve la sociedad, parece que tenemos que estar justificando lo que hacemos (PTSC).

Se le ha exigido mucho al profesorado y al alumnado. En el ámbito educativo ha habido unas exigencias muy grandes, y me parece interesante que tengan rutinas, pero revisaría la carga de tareas. (ES)

Consideran necesario capacitar al alumnado, al profesorado y a las familias en el uso de las tecnologías y dotar a los centros de los recursos necesarios.

Uno de los institutos es tecnológico y el otro no y se nota mogollón. El centro que es tecnológico ya tenía la experiencia, las aulas abiertas, los críos ya tenían su perfil, el profesorado contacta con ellos desde ahí. En el IES que es tecnológico la delegada es la que habla y comparte recursos con los grupos de clase. (ES)

Sin embargo, en los grupos en los que ya utilizaba una plataforma digital, es infinitamente más fácil porque el funcionamiento ya lo has practicado con ellos en el aula (PMAR).

De manera general, consideran que con la situación de emergencia se ha perdido la esencia de la educación, que es estar en contacto con el alumnado, con las familias y con el profesorado. Se ha perdido lo que aporta el contacto físico directo, que es cercanía, accesibilidad, complicidad y vínculo:

A través del contacto físico y la presencia despliego herramientas que me permiten comunicar. He perdido capacidad comunicativa a ese nivel, también he perdido seguridad. He perdido un lugar de confort porque las clases son para mí un lugar de confort. (VAL)

Se ha perdido el contacto directo con el alumnado, el observar. $Y$ la continuidad, el que todos los días el alumnado vaya a clase me parece algo positivo y que puedes observar muchas cosas que estando en su casa no puedes. (ES)

Una de las cosas que he descubierto es que los intercambios informales del profesorado en la cafetería, en la sala de profesores, por los pasillos son una fuente de información que es mucho más importante que lo que aparentaba ser, porque cuando eso desaparece, me he dado cuenta de que pierdo una información fundamental en el seguimiento de la situación personal de un montón de alumnos y de alumnas. Hay ciertas cosas que comentas en un pasillo "oye, échale un ojo a este que estoy preocupado, oye y esta relación que no me gusta y que tal" y todo eso se hace de una forma totalmente informal, porque no hay espacios de coordinación, pero es absolutamente fundamental. (PMAR)

Se pierde todo, el no estar con los niños y niñas, con las familias, con el profesorado, es un trabajo que ha perdido la esencia, que es la parte humana, he perdido la parte de piel, de cercanía, de accesibilidad. (OE)

Pues he perdido una parte fundamental que es la interacción persona a persona con el alumnado, el afecto, el cariño, el divertirte... el contacto con el alumnado, y con el profesorado. (PMAR)

En cuanto a las enseñanzas más destacadas del periodo de confinamiento entre las entrevistadas, destacan la importancia del trabajo en equipo, el aumento de la seguridad en una misma como profesional, la reflexión sobre la práctica docente, el ejercicio de la horizontalidad, la reconversión como profesional a distancia, aprendizajes tecnológicos y pérdida de miedos.

Perderle el miedo a equivocarme en ese sentido me he acercado al alumnado porque estamos aprendiendo mutuamente, hay una relación más horizontal así con el alumnado, me avisan de que me he equivocado en algo. (VAL) 
Se ha prestado mucha más atención a lo académico que nunca, con una visión mucho más amplia del proceso de enseñanza-aprendizaje en donde los cuidados y la inteligencia emocional se han revelado como elementos esenciales. También se ha ganado ampliamente en un "mayor seguimiento real de mi alumnado de la tutoría de cada una de sus asignaturas, he podido realmente ayudarles y empujarles a que consigan sacar el curso". (PMAR)

En un futuro será necesario actuar con una mayor seguridad y calma y mantener la política de los cuidados en sintonía con el aprendizaje educativo.

Habría que darle una pensada a los ritmos que lleva la escuela porque no son los ritmos de la vida. La escuela presencial no va al ritmo de la vida, al sacar la vida de las aulas el currículo no se sostiene con la vida de los padres, de las madres, los abuelos, las abuelas, la enfermedad..., que la escuela fuera al ritmo de la vida. Cambiaría el ritmo y valorar otras funciones educativas que no es el currículo, las fichas. (OE)

Los centros deberán tener diseñado e implantado un plan tecnológico con todas las garantías educativas ya que, durante la crisis sanitaria, se ha evidenciado la importancia de la innovación tecnológica y la educación en red.

Al mismo tiempo, las actuaciones deberán llevarse a cabo bajo criterios unificados "cada profe ha ido funcionando de una manera diferente. También en los recursos que emplean. $\Upsilon$ el alumnado ha tenido que adaptarse a cada profe". (ES)

Señalan como necesario agilizar las relaciones con los equipos docentes

El poder vernos, saber cómo están, saber qué les pasa y qué problemáticas tienen, aunque no lleguemos a acuerdos firmes, pero sí organizarnos para tener un poco de gestión interna. Trabajar en red y en equipo. Cambiaría la coordinación. La necesidad de compartir lo que nos ocurre y ponerlo en común. (VAL)

Además, se presenta una tarea fundamental desarrollar mecanismos para evitar la soledad, tanto en profesorado como en alumnado. Evitar que el trabajo individual desplace al trabajo grupal y en equipo. Será necesario reforzar el acompañamiento y los vínculos emocionales, desde la empatía, y los vínculos educativos, con una mirada integral y reformulando el currículo.

$$
\begin{aligned}
& \text { ¿Por qué tengo que seguir dando un curriculum si hay situaciones que están pasando } \\
& \text { que tiene necesidades de expresar? (VAL) }
\end{aligned}
$$

La administración educativa tendría mucha información y podría preparar a la escuela para futuras crisis, si escuchara todas las experiencias docentes y del alumnado durante este confinamiento, en un ejercicio de democracia participativa.

Creo que son fundamentales las reuniones de coordinación y creo que sería una propuesta muy positiva de cara al profesorado, a la hora de organizar su trabajo, pero creo que de cara al alumnado y a reforzar su autonomía en el aprendizaje y la generación de aprendizajes integrados, sería hacer proyectos interdisciplinares y que tengan que ver con la realidad cotidiana que están viviendo, estamos haciéndoles concentrarse en adquirir determinados contenidos, cuando su mundo se está viendo absolutamente modificado, no se habla de lo que está ocurriendo, qué pasa con la Covid, qué significa PCR, creo que proyectos contextualizados en cosas que puedan estar pasando y que les estén preocupando y que sean interdisciplinares, diría que encuentren una lógica mayor a esta pléyade de tareas. (PMAR)

Estaría guay que la administración educativa permitiera a todos los profesionales hacer una sentada para hablar de todo esto que estamos hablando nosotras "cómo lo hemos vivido, qué nos ha gustado, qué cambiariamos" para llevarlo incluso a la práctica cotidiana o estar preparadas, pero no creo que nos dejen tiempo para eso. (OE) 


\section{Discusión y conclusiones}

Se han alcanzado los objetivos planteados al principio de la investigación. A través de las entrevistas con las docentes, se han podido especificar las principales dificultades que atienden, visibilizando las desigualdades de género y señalando propuestas de cambio para situaciones similares futuras.

En relación con la situación personal y de salud, las personas entrevistadas, por regla general, no soportan excesivas cargas familiares (solo una de ellas tiene personas dependientes a su cargo) y, pese a ello, señalan estar agotadas y afectadas en su salud mental. Es fácil pensar que si esta situación fuera otra, si las entrevistadas hubieran tenido mayores cargas familiares, los resultados arrojados en las entrevistas habrían sido todavía más devastadores.

Resulta contradictorio que, aunque se identifican de manera tenue algunos problemas derivados del sedentarismo y de pasar muchas horas delante del ordenador, no existe una comunicación de la salud desde una visión integral, entiendo el bienestar en la salud desde un paradigma biopsicosocial. Sin embargo y a pesar del malestar manifiesto a todos los niveles de salud, ni las profesionales entrevistadas ni la población en general, identifican como problemas de salud dichas manifestaciones, aunque la OMS prevé que uno de las grandes dificultades tras la pandemia serán los trastornos psíquicos y emocionales (EFE, 2020).

En relación con la situación laboral, se ha entrevistado a personas con condiciones laborales estables, pertenecientes a la Comunidad de Madrid, estrechamente vinculadas con labores de orientación y tutoría por lo que sus respuestas han estado determinadas por una clara conciencia social y compromiso educativo. Son profesionales que pertenecen a la escuela pública, con una mayoría de alumnado a su cargo con dificultades sociales y económicas, en ocasiones, con riesgo de absentismo y poca motivación por el estudio y grandes dificultades de conexión a Internet y de tenencia de dispositivos. Probablemente si las entrevistas se hubieran realizado a profesionales de la privada o la concertada, los resultados hubieran variado (Molina-Pérez y Luengo, 2020).

En general el tiempo de trabajo ha tomado las casas, los espacios familiares, el tiempo libre y los fines de semana. Las profesionales entrevistadas se ven obligadas a una conexión permanente, sin limitación horaria y horarios condicionados por las familias del alumnado. Los recursos utilizados son los propios (conexiones a Internet, ordenadores, teléfonos móviles) en lugares de trabajo, como son sus propias residencias, sin preparar para un trabajo online de estas características, irrumpiendo en su propia vida familiar con las consecuencias que esto puede conllevar.

No es difícil pensar en cómo puede ser su vida, conectadas de manera permanente por medio de videoconferencias, de video llamadas por WhatsApp y llamadas telefónicas, explicando temas, atendiendo a las realidades de su alumnado, metiendo dentro de sus casas respectivas a un montón de gente, sin un horario fijo. Mucho más aun en una situación de estado de alarma en donde los límites y las fronteras han quedado difuminados ¿Repercutirá en sus relaciones familiares, en sus relaciones de pareja, en su salud mental? La respuesta resulta evidente.

En lo relativo a la labor docente, han sido patentes dos periodos claramente diferenciados, que se corresponden con antes y después de las vacaciones de semana santa, asignadas en el calendario escolar. Los objetivos pasaron de ser más emocionales a ser más académicos. 
En un primer momento era importante localizar al alumnado, ponerse a su disposición y a la de las familias, y capacitarles para enfrentarse a la enseñanza online. Todo sin una coordinación institucional y asumiendo una nueva situación personal y laboral. La segunda fase, coincide con el comienzo de instrucciones más claras por parte de la Consejería de Educación, lo que permitió ir readaptando los objetivos iníciales y abordar la parte más académica, pero sin dejar de hacer seguimiento desde la parte más afectiva, para lograr mantener el vínculo con el alumnado. Es decir, la educación no es solo instrucción, como muchas veces se hace ver, el proceso educativo va mucho más allá de los contenidos que se transmiten de manera obligatoria (Pumares y Salazar, 2007). Ha tenido que llegar una situación de emergencia social para comprobar la labor de cuidado que desarrolla el profesorado y la importancia de los vínculos afectivos generados presencialmente.

Como problemática general en el proceso de enseñanza durante el confinamiento, se alude a la falta de coordinación en los centros escolares. No es muy fácil ver una cultura de lo colectivo en el ámbito educativo (Belavi y Murillo, 2020) y una coordinación horizontal. Es por ello, que la soledad haya destacada como algo característico de los profesionales de la educación durante el confinamiento.

En cuanto a los recursos que les han sido útiles, aluden a algo relacionado con lo anterior: ponerse en contacto personalmente con familias y alumnado, mantener el contacto personal (por medio de llamadas y WhatsApp desde sus propios teléfonos o videoconferencias en las que la clase pudiera verse, desde sus respetivos ordenadores) e intentar mantener el ánimo y la motivación por estas vías, así como permitir que el alumnado pudiera contarles su estado personal. También aluden a recursos virtuales proporcionados por la Comunidad de Madrid o, incluso, instituciones privadas. Es un hecho que, sin su labor mediadora y su esfuerzo e implicación personal con el alumnado, la efectividad en la educación en confinamiento no hubiera tenido el mismo impacto.

Consideran, a futuro cercano, que el curso que entra será complicado. Se manejan con la incertidumbre de cómo va a ser y, lo más importante, será evidente que habrá que manejar psicológicamente las pérdidas de familiares, la pérdida de trabajos en las familias del alumnado y la afectación emocional de alumnas y alumnos. Sería preciso poder dedicar tiempo a reparar las situaciones traumáticas vividas. Sería, por lo tanto, un buen momento para dejar de lado esos currículos cargados de contenidos conceptuales, que no dan margen para la innovación y sin espacio posible para dedicarlo a lo personal (Sánchez Sáinz, 2019).

Se habla de la brecha digital pero no es tan habitual visibilizar la brecha de género. Si ya viene siendo un tema menor (Ballester, 2018), no es de extrañar que se haya manifestado aún más durante el confinamiento. Los resultados evidencian que las labores de cuidados han continuado en manos de las mujeres (tanto profesoras como alumnas) y las labores del espacio público y a la utilización de la tecnología siguen en manos de los hombres.

Si a esto le unimos que la falta de la presencialidad ha complicado la detección de posibles violencias y que, eliminando el espacio escolar, se elimina la posibilidad de equidad en muchos de los casos, podemos encontrarnos con un serio problema a este respecto.

Hay que señalar que las personas entrevistadas son todas mujeres y con una perspectiva feminista. Ellas han sido capaces de ver indicios que, tal vez, otras personas no. Por ello resultaría interesante hacer estudios más pormenorizados sobre estas cuestiones, 
analizando, por ejemplo, cómo ha sido el rendimiento académico de las niñas que han vivido alternativamente con su padre o con su madre, si ha habido violencias que no se hayan detectado, quién ha tenido un mayor acceso a la tecnología en las casas o quién ha sostenido los cuidados básicos durante el confinamiento.

En relación con los aprendizajes que ha traído la situación de confinamiento, en caso de que esta se repitiera, es destacable la sensación docente de tener que justificar permanentemente su trabajo y su salario, lo que ha llevado a incrementar más si cabe, su horario laboral y a mandar tareas al alumnado, a veces de manera indiscriminada y poco coordinada. Se encuentran fuertemente instalados en la sociedad la sospecha y el prejuicio frente al trabajo docente, exigiendo una demostración laboral permanente para rendir cuentas a las familias, a la sociedad en su conjunto y, como no, a la Administración Educativa (Zafra, 2017).

Resulta evidente que, con la enseñanza online, se ha perdido la esencia de la educación en sí misma, la piel, las miradas, la complicidad... los vínculos entre docentes, entre alumnado y entre unos y otros. Por eso cuando se afirma desde los medios de comunicación que la educación online ha venido para quedarse, se demuestra una gran ignorancia de lo que es la educación en sí misma como algo que nada o muy poco tiene que ver con la mera instrucción y sí con la afectividad como eje de acción (Cariño et al.,2017).

No obstante, sí que ha habido aprendizajes en estos meses, tales como hacer patente la importancia del trabajo en equipo y los cuidados (de nosotras mismas y del grupo), reflexionar sobre nuestra propia práctica y la posibilidad de equivocarnos, o poner en tela de juicio el sistema educativo y el currículo sobrecargado que solo transmite contenidos conceptuales.

Como ideas para futuras situaciones similares y, también aplicables a la enseñanza presencial, son destacables:

- Actuar con más calma ya que la escuela no puede estar al margen de los ritmos de la vida.

- Aplicar la política de los cuidados al aula, a los grupos docentes, al centro educativo.

- Agilizar las relaciones y la coordinación entre los equipos docentes y establecer criterios unificados de acción.

- Evitar que la individualidad desplace a la colectividad.

- Dar importancia al acompañamiento emocional desde una mirada educativa integral.

Cuestiones que no eximen a la Administración Educativa de diseñar e implementar un plan tecnológico con garantías. Entendemos que la Administración Educativa tiene una enorme responsabilidad de cara al funcionamiento futuro, no tanto burocratizando la educación sino escuchando a las y los docentes, al alumnado y a sus familias. Esto que hemos intentado hacer en estas páginas, dar voz a las personas implicadas, debería hacerlo la administración, en un ejercicio de democracia real que implique la participación de la comunidad educativa en su conjunto.

$\mathrm{Si}$ algo ha dejado claro esta situación de confinamiento es que los modelos educativos individualistas y basados en la acumulación y reproducción de contenidos tienen que 
quedar en el pasado y que debemos pensar otras formas de hacer educación en sintonía con la vida del alumnado (Vergara, 2020). Es evidente que el sistema educativo actual está en crisis, que hace falta pensar desde otros lugares, buscar puntos de fuga (Flores, 2018) para poder estar más en línea con la vida.

En otra línea de paralelismos podemos suponer, siguiendo a Preciado (2020), que al igual que la pandemia del VIH cambió las relaciones sexuales y la educación sexual, la pandemia de la Covid-19 cambiará las relaciones sociales y educativas. Si esto ocurre, ¿cómo deberá educar la escuela a la futura ciudadanía?

Mientras los cambios llegan deberemos tomar esta nueva forma de educar, más que como un problema, como un reto, como una oportunidad de generar nuevos aprendizajes por parte de todas las personas implicadas. Teniendo muy claro que la enseñanza online nunca podrá sustituir a la educación presencial porque sería obviar el carácter afectivo de la educación (Hooks, 1994). Sería obviar que la escuela es un espacio de equidad y de igualdad de oportunidades, que dentro de la escuela se previenen y detectan muchos tipos de violencias, en definitiva, que la escuela no solo educa, también cuida.

Otra cosa es que haya que repensar la escuela y sus formas, que haya que repensar el currículo y las políticas educativas, para hacer que sean más acordes con la vida y nuestras aspiraciones como sociedad (Belavi y Murillo 2020). Habría que dar voz al profesorado, hacer partícipes a todos los miembros de la comunidad educativa, para lograr una escuela participativa y democrática. Sería urgente idear una escuela desde los afectos, los cuidados y la creatividad, hacer de la escuela un espacio de resistencia (Moreno y Penna, 2018) para poder cambiar el modelo de ciudadanía que queremos y, tal vez, poder cambiar el curso de la historia, ayudando a que otra educación y otro mundo sean posibles (Torres, 2017).

Gracias a todas las profesionales que nos prestaron su tiempo, en una situación de alarma, de preocupación y de agotamiento, para poder entender lo que está pasando e imaginar un futuro mejor.

\section{Referencias}

Almazán, A. (2020). Covid-19: ¿Punto sin retorno de la digitalización de la educación? Revista Internacional de Educación para la Justicia Social, 9(3e).

Anzaldúa, G. (2004). Los movimientos de rebeldía y las culturas que traicionan. En C. Romero y S. García Dauder (Coords.), Otras inapropiables. Feminismos desde la frontera (pp. 71-80). Traficantes de sueños.

Ballester, M. A. (19 de febrero de 2018). Lo que puede hacer el parlamento para corregir la desigualdad retributiva entre mujeres y hombres. Eldiario.es. https://www.eldiario.es/autores/maria_amparo_ballester/

Belavi. G. y Murillo, F. J. (2020). Democracia y justicia social en las escuelas: Dimensiones para pensar y mejorar la práctica educativa. REICE. Revista Iberoamericana sobre Calidad, Eficacia y Cambio en Educación, 18(3), 5-28. https://doi.org/10.15366/reice2020.18.3.001

Burgess, S. y Sievertsen. H. H. (19 de mayo de 2020). Schools, skills, and learning: The impact of Covid-19 on education. VOX CEPR Policy Portal. https://voxeu.org/article/impact-Covid19-education

Cariño, C., Cumes, A., Curiel, O., Garzón, M. T., Mendoza, B., Ochoa, K. y Londoño, A. (2017). Pensar, sentir y hacer pedagogías feministas descoloniales: Diálogos y puntadas. En C. 
Walsh (Ed.), Pedagogías decoloniales: Prácticas insurgentes de resistir, (re)existir y (re)vivir. Ediciones Abya-Yala

Ceballos, N. A. y Sevilla, S. (2020). El efecto del aislamiento social por el Covid-19 en la conciencia emocional y en la comprensión lectora. Estudio sobre la incidencia en alumnos con trastornos de aprendizaje y menor acceso a las nuevas tecnologías. Revista Internacional de Educación para la Justicia Social, 9(3e), 7-23.

Cejudo, C. M. A. y Corchuelo, C. (2019). La prevención del VIH/SIDA en la formación universitaria de los futuros educadores. Revista de Humanidades, 37, 127-150. https://doi.org/ 10.5944/rdh.37.2019.20060

Cervantes, C. y Gutiérrez, P. R. (2020). Resistir la Covid-19. Intersecciones en la educación de Ciudad Juárez, México. Revista Internacional de Educación para la Justicia Social, 9(3e), 7-23.

Comunidad de Madrid. (30 de marzo de 2020). El 90\% de los alumnos de bachillerato de la Comunidad de Madrid cumple con sus trabajos online. Comunidad de Madrid. https://www.comunidad.madrid/noticias/2020/03/30/90-alumnos-bachilleratocomunidad-madrid-cumple-trabajos-online

Cooper, H., Borman, G. y Fairchild, R. (2010). School calendars and academic achievement. En J. L. Meece (Ed.), Handbook of research on schools, schooling, and human development. Routledge. https:///doi.org/10.4324/9780203874844

Corres-Medrano, I. y Santamaria-Goicuria, I. (2020). Miedos en una sociedad enferma. Revista Internacional de Educación para la Justicia Social, 9(3e).

https://revistas.uam.es/riejs/article/view/12121/12018

De Alonso, A. (2020). Contra el virus de la vulnerabilidad. Revista Internacional de Educación para la Justicia Social, 9(3e). https://revistas.uam.es/riejs/article/view/12 105/12015

Díez-Gutiérrez, E. J. (21 de abril de 2020). Educación lenta: Menos es más. El Diario de la Educación. https://eldiariodelaeducacion.com/2020/04/21/educacion-lenta-menos-esmas/

EFE. (14 de mayo de 2020). La OMS advierte que el coronavirus afectará a nuestra salud mental futura. El Confidencial. https://www.elconfidencial.com/mundo/2020-05-14/coronavirusoms-nuestra-salud-mental-futura_2594312/

Fernández Savater, A. (16 de marzo de 2020). Habitar la excepción: Pensamientos sin cuarentena. Filosofía Pirata. https://www.filosofiapirata.net/habitar-la-excepcion-pensamientos-sincuarentena-i/

Flores, V. (2013). Interrupciones. Ensayos de poética activista. Escritura, política, educación. La Mondonga Dark.

Gárate, F. (2020). Desde la injusticia social a la injusticia educativa o desde la injusticia social a la escuela como protagonista de la transformación educativa y social. Revista Internacional de Educación para la Justicia Social, 9(3e).

García Medina, R., Penna, M., Sánchez, M., Salguero, J. M. y Moreno, I. (2020). Análisis de los itinerarios de éxito de estudiantes migrantes y estudiantes trans que alcanzaron estudios universitarios, desde una perspectiva educativa inclusiva. Revista Complutense de Educación, 31(2), 207-218. https://doi.org/10.5209/rced.62016

Gil, S. L. (8 de mayo de 2020). Amigas, ¿a qué mundo queremos regresar? Ctxt y acción. https://ctxt.es/es/20200501/Firmas/32177/Silvia-Gil-feminismo-coronavirus-mundonormalidad-cuidados-capitalismo.htm 
Gómez Gerdel, M. A. (2020). El cerebro pleno del niño/a: La labor de un/a maestro/a de educación inclusiva con las familias en tiempos de confinamiento. Una reflexión educativa. Revista Internacional de Educación para la Justicia Social, 9(3e). https://revistas.uam.es/riejs/article/view/12095/12012

Hodges, C., Moore, S., Lockee, B., Trust, T. y Bond, A. (27 de marzo de 2020). The difference between emergency remote teaching and online learning. EducauseReview. https://bit.ly/3cng48F

Hooks, B. (1994). Teaching to transgress: Education as the practice of freedom. Routledge. https://doi.org/10.3366/para.1994.17.3.270

INEE. (2019). PISA 2018. Programa para la Evaluación internacional de los estudiantes informe español. Ministerio de Educación y Formación Profesional.

Gascón, M. (29 de abril de 2020). Los efectos del coronavirus en la educación: la brecha digital ya deja atrás a millones de estudiantes. 20 Minutos. https://www.20minutos.es/noticia/4241143/o/los-efectos-del-coronavirus-en-laeducacion-la-brecha-digital-ya-deja-atras-a-millones-de-estudiantes /

Juárez, J. M., de Oña, J. M. y Molina, L. (2020). Vivir el Covid-19 en una chabola: Resiliencia en situaciones de desigualdad social. Revista Internacional de Educación para la Justicia Social, $9(3 \mathrm{e})$.

Ministerio de Igualdad. (2020). La perspectiva de género, esencial en la respuesta a la Covid-19. Instituto de la Mujer.

Molina-Pérez, J. y Luengo, J. (2020). Reconstrucciones “resilientes” de la identidad profesional del profesorado: Endoprivatización y cultura performativa en Andalucía (España). REICE. Revista Iberoamericana sobre Calidad, Eficacia y Cambio en Educación, 18(2), 57-75. https://doi.org/10.15366/reice2020.18.2.003

Moreno, D. (5 de mayo de 2020). Las élites utilizan la crisis del Covid-19 para avanzar en su excluyente hoja de ruta. El Salto. https://www.elsaltodiario.com/coronavirus/entrevistanaomi-klein-gente-habla-volver-normalidad-crisis-doctrina-shock

Moreno, Y. y Penna, M. (2018). Breve decálogo de ideas para una escuela feminista. Revista Trabajadoras de la Enseñanza.

Muñoz, J. L. y Lluch, L. (2020). Educación y Covid-19: Colaboración de las familias y tareas escolares. Revista Internacional de Educación para la Justicia Social, 9(3e).

OMS. (2020). Coronavirus disease 2019 (Covid-19). Situation report-100. OMS.

Pecharromán, C. (19 de mayo de 2020). Las peticiones de ayuda por violencia de género se disparan un $61 \%$ durante el estado de alarma. RTVE.

https://www.rtve.es/noticias/20200519/peticiones-ayuda-violencia-genero-se-disparan61-durante-estado-alarma/2014382.shtml

Peñate, L. M. (2020). Todos somos importantes, pero el docente es imprescindible. Revista Internacional de Educación para la Justicia Social, 9(3e).

Preciado, P. (14 de abril de 2020). Aprendiendo del virus. Sexuality Policy Watch. https://sxpolitics.org/es/paul-b-preciado-aprendiendo-del-virus / 4740

Pumares, L. y Salazar, J. (2007). Quitando cárcel a la escuela. CEP.

Rodríguez Morales, P. (2020). ¿Políticas uniformes y universales de acceso a la tecnología revierten desigualdades educativas? Revista Internacional de Educación para la Justicia Social, 9(3e). 
Ruiz-Valenzuela, J. (2020). Intergenerational effects of employment protection reforms. Labour Economics, 62, 13-28. https://doi.org/10.1016/j.labeco.2019.101774

Sánchez Sáinz, M. (2019). Pedagogías queer ¿̇nos arriesgamos a hacer otra educación? La Catarata.

Sáinz, J. y Sanz, I. (23 de abril de 2020). Los efectos del ccoronavirus en la educación: Las pérdidas de clases y rendimientos educativos desiguales. Nada es gratis.

https://nadaesgratis.es/admin/los-efectos-del-coronavirus-en-la-educacion-i-lasperdidas-de-clases-y-rendimientos-educativos-desiguales

Sandoval, C. (2004). Nuevas ciencias, feminismos ciborg y metodología de los oprimidos. En C. Romero y S. García Dauder (Coords.), Otras inapropiables. Feminismos desde la frontera. Traficantes de sueños.

Santos, B. D. S. (2020). La cruel pedagogía del virus. CLACSO.

Simón, E. (2010). La igualdad también se aprende: Cuestión de coeducación. Narcea.

Torres, J. (2017). Políticas educativas y construcción de personalidades neoliberales y neocolonialistas. Morata.

Vergara, J. J. (2020). O se llama coravid o no existe. Revista Internacional de Educación para la Justicia Social, 9(3).

Zafra, R. (2017). El entusiasmo. Anagrama.

\section{Breve CV de las autoras}

\section{Melani Penna Tosso}

Psicóloga, doctora en educación. Es profesora y escritora. Es Co-directora del posgrado en Pedagogías Feministas y Queer, título propio de la Universidad Complutense y docente en el Máster de Estudios LGBTQ+ de la misma universidad en la que trabaja como Personal Docente e Investigador dentro del Departamento de Estudios. Miembro del grupo consolidado de investigación INDICES. Ha ejercido como profesora en las diferentes etapas educativas en diferentes países dirigiendo durante cuatro años el Proyecto de Investigación PESI: Programa de Educación Sexual Integral, financiado por el Ministerio de Educación de Ecuador. Autora de libros, artículos y materiales educativos. Como escritora tiene publicado un libro de cuentos "El mundo raro de Mermel" y en el año 2021 se publicará su primera novela con la editorial Dos Bigotes. ORCID ID: https://orcid.org/o0o0-0002-82 11-8664. Email: melani.penna@edu.ucm.es

\section{Mercedes Sánchez Sáinz.}

Doctora en educación. Pedagoga. Logoterapeuta infantil. Profesora de la Facultad de Educación de la Universidad Complutense de Madrid desde 1999. Ha sido docente en Primaria, Secundaria y Universidad. Actualmente impartiendo asignaturas de equidad e inclusión a Educación Social, Magisterio, Pedagogía y Másteres. Autora de múltiples publicaciones relacionados con la diversidad sexual y las identidades de género en ámbito educativo. 49 trabajos publicados (229 citaciones, índice h 7 , índice i10 6). Co-creadora de la primera oficina de diversidad sexual e identidad de género en una universidad española. Forma parte de la comisión académica del primer máster oficial de Estudios LGBTIQ+ en territorio español. Líneas de investigación: inclusión y diversidad sexual e identidad de género en ámbito educativo. Participación en 16 proyectos de investigación. Miembro del 
grupo consolidado UCM de investigación INDICES. 2 tesis dirigidas y defendidas y otras dos en proceso. Participación en 5 PIMCD y coordinación de uno. ORCID ID: https://orcid.org/0000-0002-7547-4724. Email: mercesan@edu.ucm.es

\section{Cristina Mateos Casado}

Doctora especializada en violencia de género por la Universidad Complutense de Madrid. Trabajadora Social y Socióloga con especialidad en análisis político. En la actualidad, es profesora asociada en el Departamento de Periodismo y Comunicación Global de la Universidad Complutense de Madrid. Trabajó como docente investigadora (2012-2016) en las Facultades de Filosofía, Letras y Ciencias de la Educación y Ciencias Humanísticas y Sociales de la Universidad Técnica de Manabí, Ecuador. En el ámbito social ha trabajado como trabajadora social y consultora especializada en violencia de género y en migraciones en diferentes organizaciones e instituciones de la Comunidad de Madrid y Castilla y León. Ha publicado artículos científicos en revistas de impacto, guías técnicas, protocolos para la intervención social, y colaborado en la revisión y edición de libros. ORDIC ID: https://orcid.org/o000-0001-9813-7491. Email: cmate01@ucm.es 